\title{
Nonequilibrium dynamics in an interacting Fe-C nanoparticle system
}

\author{
Jönsson, P.; Hansen, Mikkel Fougt; Nordblad, P.
}

Published in:

Physical Review B Condensed Matter

Link to article, DOI:

10.1103/PhysRevB.61.1261

Publication date:

2000

Document Version

Publisher's PDF, also known as Version of record

Link back to DTU Orbit

Citation (APA):

Jönsson, P., Hansen, M. F., \& Nordblad, P. (2000). Nonequilibrium dynamics in an interacting Fe-C nanoparticle system. Physical Review B Condensed Matter, 61(2), 1261-1266. https://doi.org/10.1103/PhysRevB.61.1261

\section{General rights}

Copyright and moral rights for the publications made accessible in the public portal are retained by the authors and/or other copyright owners and it is a condition of accessing publications that users recognise and abide by the legal requirements associated with these rights.

- Users may download and print one copy of any publication from the public portal for the purpose of private study or research.

- You may not further distribute the material or use it for any profit-making activity or commercial gain

- You may freely distribute the URL identifying the publication in the public portal

If you believe that this document breaches copyright please contact us providing details, and we will remove access to the work immediately and investigate your claim 


\title{
Nonequilibrium dynamics in an interacting Fe-C nanoparticle system
}

\author{
P. Jönsson \\ Department of Materials Science, Uppsala University, Box 534, SE-751 21 Uppsala, Sweden \\ M. F. Hansen \\ Department of Materials Science, Uppsala University, Box 534, SE-751 21 Uppsala, Sweden \\ and Department of Physics, Building 307, Technical University of Denmark, DK-2800 Lyngby, Denmark \\ P. Nordblad \\ Department of Materials Science, Uppsala University, Box 534, SE-751 21 Uppsala, Sweden
}

(Received 3 September 1999)

\begin{abstract}
Nonequilibrium dynamics in an interacting $\mathrm{Fe}-\mathrm{C}$ nanoparticle sample, exhibiting a low-temperature spinglass-like phase, has been studied by low-frequency ac susceptibility and magnetic relaxation experiments. The nonequilibrium behavior shows characteristic spin-glass features, but some qualitative differences exist. The nature of these differences is discussed.
\end{abstract}

\section{INTRODUCTION}

The dynamics of interacting nanoparticle systems has been subject of considerable interest concerning the existence of a low-temperature spin-glass phase. Evidence for a phase transition from a high-temperature superparamagnetic phase to a low-temperature spin-glass-like phase has been given from reports on critical slowing down ${ }^{1}$ and a divergent behavior of the nonlinear susceptibility. ${ }^{2,3}$

Spin glasses have been intensively investigated during the two last decades, not only concerning the phase transition but also the nature of the spin-glass phase. The nonequilibrium dynamics in the spin-glass phase has been extensively studied by conventional and also more sophisticated dcmagnetic relaxation and low-frequency ac-susceptibility experiments. ${ }^{4}$ A nonequilibrium character of the lowtemperature spin-glass-like phase of interacting particle systems has been revealed from measurements of dc-magnetic relaxation $^{1,5}$ and relaxation of the low-frequency ac susceptibility. ${ }^{6}$ In this paper, the nonequilibrium dynamics in the spin-glass-like phase is further elucidated and special interest is put into a dynamic memory effect, which has recently been observed in spin glasses. ${ }^{7-10}$ In a "memory experiment" the ac susceptibility is measured at a low frequency, and the cooling of the spin-glass sample is halted at one (or more) temperatures. During a halt, $\chi$ slowly decays, but when cooling is resumed $\chi$ gradually regains the amplitude of a continuous cooling experiment. Upon the following heating, $\chi$ shows dips at the temperatures where the temporary halts occurred; a memory of the cooling history has been imprinted in the spin structure. (For an illustration, see Fig. 1 in Ref. 8.) A similar memory of the cooling process has recently been reported in an interacting nanoparticle system. ${ }^{11}$

\section{THEORETICAL BACKGROUND}

For spin glasses, the nonequilibrium dynamics has been interpreted within a phenomenological real-space model, ${ }^{8}$ adopting important concepts from the droplet model. ${ }^{12} \mathrm{We}$ will use the same real-space model to discuss similarities and dissimilarities between the low-temperature phase of interacting particle systems and that of spin glasses.

The droplet model was derived for a short-range Ising spin glass, but important concepts like domain growth, chaos with temperature, and overlap length should be applicable also for particle systems exhibiting strong dipole-dipole interaction and random orientations of the anisotropy axes. Chaos with temperature means that a small temperature shift changes the equilibrium configuration of the magnetic moments completely on sufficiently long length scales. The length scale, up to which no essential change in configuration of the equilibrium state is observed after a temperature step $\Delta T$, is called the overlap length $l(\Delta T)$. The development towards equilibrium is governed by the growth of equilibrium domains. The typical domain size after a time $t_{\mathrm{w}}$ at a constant temperature $T$ is

$$
R\left(T, t_{\mathrm{w}}\right) \propto\left(\frac{T \ln \left(t_{\mathrm{w}} / \tau\right)}{\Delta(T)}\right)^{1 / \psi},
$$

where $\tau$ is the relaxation time of an individual magnetic moment, $\Delta(T)$ sets the free-energy scale and $\psi$ is a barrier exponent. For spin glasses, the atomic relaxation time is of the order of $10^{-13} \mathrm{~s}$ independent of temperature, while for magnetic particles, the individual particle relaxation time is given by an Arrhenius law as

$$
\tau=\tau_{0} \exp \left(\frac{K V}{k_{B} T}\right),
$$

where $\tau_{0} \sim 10^{-12}-10^{-9} \mathrm{~s}, K$ is an anisotropy constant, $V$ the particle volume, and thus $K V$ the anisotropy energy barrier. Due to the inevitable polydispersivity of particle systems the individual particle relaxation time will also be distributed, and due to the exponential factor this distribution will be broader than the distribution of anisotropy energy barriers.

In the droplet model there exists only two degenerate equilibrium spin configurations, $\Psi$ and its spin-reversal 
counterpart $\widetilde{\Psi}$. It is thus possible to map all spins to either of the two equilibrium configurations. Let us now consider a spin glass quenched to a temperature $T_{1}$ lower than the spinglass transition temperature $T_{g}$. At $t=0$ the spins will randomly belong to either of the two equilibrium states, resulting in fractal domains of many sizes. The subsequent equilibration process at $t>0$ is governed by droplet excitations yielding domain growth to a typical length scale, which depends on temperature and wait time $t_{\mathrm{w}_{1}}$ according to Eq. (1). After this time, fractal structures typically smaller than $R\left(T_{1}, t_{\mathrm{w}_{1}}\right)$ have become equilibrated while structures on longer length scales persist. If the system thereafter is quenched to a lower temperature $T_{2}$, the spins can be mapped to the equilibrium configuration at $T_{2}$ yielding a new fractal domain structure. The domain structure achieved at temperature $T_{1}$ still fits to the equilibrium configuration at $T_{2}$ on length scales smaller than the overlap length $l\left(T_{1}-T_{2}\right)$. The domain growth at $T_{2}$ will start at the overlap length, increase with wait time $t_{\mathrm{w}_{2}}$, and end up at the size $R\left(T_{2}, t_{\mathrm{w}_{2}}\right)$. Heating the system back to $T_{1}$, the fractal domain growth that occurred at $T_{2}$ has only introduced a new and dispersed domain structure at $T_{1}$ on length scales $l\left(T_{1}-T_{2}\right) \leqslant R$ $\leqslant R\left(T_{2}, t_{\mathrm{w}_{2}}\right)$. Note that the large length scale structures, $R$ $\geqslant R\left(T_{1}, t_{\mathrm{w}_{1}}\right)$, essentially persist.

The time-dependent response $m\left(t_{\mathrm{obs}}\right)$ to a weak magnetic field applied at $t_{\mathrm{obs}}=0$, is due to a continuous magnetization process, governed by polarization of droplets of size

$$
L\left(T, t_{\mathrm{obs}}\right) \propto\left(\frac{T \ln \left(t_{\mathrm{obs}} / \tau\right)}{\Delta(T)}\right)^{1 / \psi} .
$$

Since $L\left(T, t_{\mathrm{obs}}\right)$ grows with the same logarithmic rate as $R\left(T, t_{\mathrm{w}}\right)$, the relevant droplet excitations and the actual domain sizes become comparably large at time scales $\ln t_{\mathrm{obs}}$ $\approx \ln t_{\mathrm{w}}$. For $\ln t_{\mathrm{obs}} \ll \ln t_{\mathrm{w}}$ the relevant excitations occur mainly within equilibrated regions, while for $\ln t_{\mathrm{obs}} \gg \ln t_{\mathrm{w}}$ these excitations occur on length scales of the order of the growing domain size, and involves domain walls, yielding a nonequilibrium response. A crossover from equilibrium to nonequilibrium dynamics occurs for $\ln t_{\mathrm{obs}} \approx \ln t_{\mathrm{w}}$, seen as a maximum (or only a bump) in the relaxation rate $S(t)$ $=h^{-1} \partial m(t) / \partial \ln t$ vs $\ln t$ curves.

dc-relaxation and ac-susceptibility experiments are related through the relations $M(t) \approx \chi^{\prime}(\omega)$ and $S(t) \propto \chi^{\prime \prime}(\omega)$, when $t=1 / \omega .{ }^{13}$ Hence the aging behavior is also observed in lowfrequency ac-susceptibility measurements. Different from dc-relaxation measurements is that the observation time is constant, $t_{\mathrm{obs}}=1 / \omega$, implying that the probing length scale $L(T, 1 / \omega)$ is fixed for a given temperature.

\section{EXPERIMENT}

The sample consisted of ferromagnetic particles of the amorphous alloy $\mathrm{Fe}_{1-x} \mathrm{C}_{x}(x \approx 0.22)$ prepared by thermal decomposition of $\mathrm{Fe}(\mathrm{CO})_{5}$ in an organic liquid (decalin) in the presence of surfactant molecules (oleic acid) as described in Ref. 14. After the preparation, the carrier liquid was evaporated and the particles were transferred to xylene in an oxygen-free environment resulting in a ferrofluid with a particle concentration of $5 \mathrm{vol} \%$ (determined by atomic absorp-

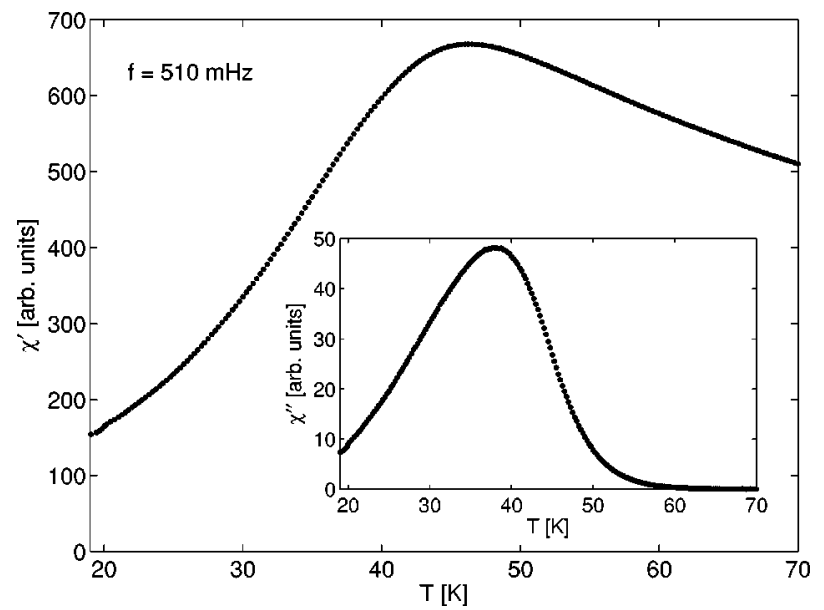

FIG. 1. The ac susceptibility vs temperature at $f=510 \mathrm{mHz}$.

tion spectroscopy). The particles were separated by the surfactant coating and could only interact via magnetic dipoledipole interactions. During measurements, the ferrofluid was contained in a small sapphire cup sealed with epoxy glue to prevent oxidation of the particles. The sample was only measured and exposed to magnetic fields well below the melting point of xylene $(\approx 248 \mathrm{~K})$. A droplet of the ferrofluid was dripped onto a grid for transmission electron microscopy and was left to oxidize prior to the study. The electron micrographs revealed a nearly spherical particle shape. The volume-weighted size distribution (after correction for the change in density due to oxidation) was described well by the log-normal distribution,

$$
f(V) d V=\left(\sqrt{2 \pi} \sigma_{V} V\right)^{-1} \exp \left[-\ln ^{2}\left(V / V_{m}\right) /\left(2 \sigma_{V}^{2}\right)\right] d V,
$$

with the median volume $V_{m}=8.6 \times 10^{-26} \mathrm{~m}^{3}$ and logarithmic standard deviation $\sigma_{V}=0.19$. These parameters correspond to a log-normal volume-weighted distribution of particle diameters with the median value $d_{m}=\left(6 V_{m} / \pi\right)^{1 / 3}$ $=5.5 \mathrm{~nm}$ and $\sigma_{d}=\sigma_{V} / 3=0.062$. This particle size is slightly larger and the value of $\sigma_{V}$ is slightly lower than those reported in recent studies ${ }^{1,2,15}$ performed on a different batch of particles. The $5 \mathrm{vol} \%$ sample has been subject to a detailed study of the correlations and magnetic dynamics using a wide range of observation times and temperatures. ${ }^{16}$ Collective behavior is observed at temperatures below $40 \mathrm{~K}$.

A noncommercial low-field superconducting quantum interference device magnetometer ${ }^{17}$ was used for the measurements. All ac susceptibility measurements were performed at a frequency of $510 \mathrm{mHz}$ and a rms value of the ac field of $0.1 \mathrm{Oe}$. The cooling and heating rate was $0.25 \mathrm{~K} / \mathrm{min}$. For the relaxation measurements the dc field was $0.05 \mathrm{Oe}$, while the background field was less than $1 \mathrm{mOe}$.

\section{RESULTS AND DISCUSSION}

\section{A. General behavior}

Figure 1 shows the ac susceptibility of the particle sample measured while cooling. The onset of the out-of-phase component of the susceptibility is less sharp than for spin glasses. Nevertheless, there exists collective spin-glass-like dynamics in the particle system, which can be studied by magnetic aging experiments. The "conventional" aging experiment is 


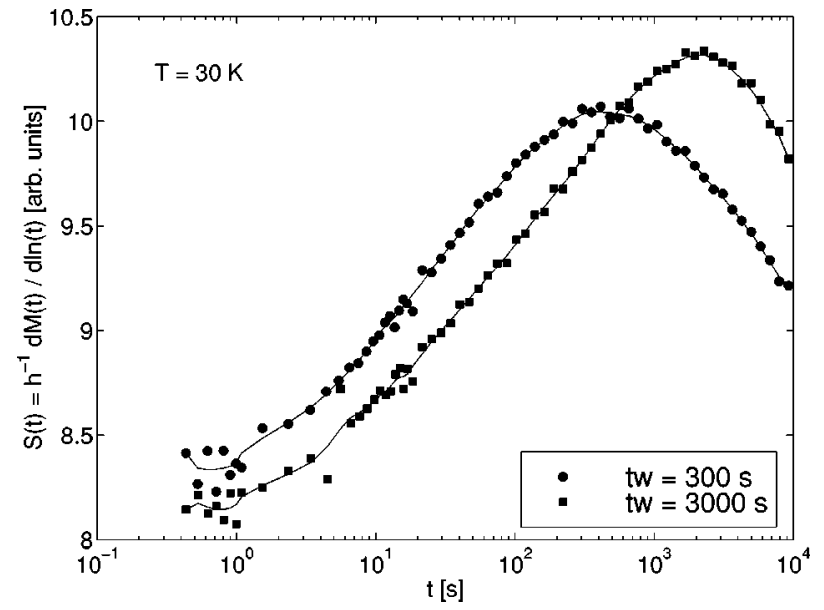

FIG. 2. The relaxation rate of the zero-field-cooled magnetization vs time at $T=30 \mathrm{~K}$. The wait time is $300 \mathrm{~s}$ (circles) and 3000 s (squares).

a measurement of the wait time dependence of the response to a field change, after either field cooling (thermoremanent magnetization, TRM) or zero-field cooling (ZFC). In Fig. 2, the relaxation rate from two $\mathrm{ZFC}$ magnetic relaxation measurements is shown. The particle system was directly cooled from a high temperature to $30 \mathrm{~K}$ where it was kept a wait time of 300 and $3000 \mathrm{~s}$, respectively, before the magnetic field was applied. A clear wait time dependence is seen; the relaxation rate displays a maximum at $\ln t_{\mathrm{obs}} \approx \ln t_{\mathrm{w}}$. Both particle systems and spin glasses exhibit magnetic aging that affects the response function in a similar way, but the wait time dependence appears weaker for the particle system. Comparing the ratio between the magnitude of $S(t)$ at the maximum and at the short time limit $(0.3 \mathrm{~s}$ in our current experiment) we find a value of order 1.3 in the current sample, whereas most spin glasses would show a value $\geqslant 2$, at a corresponding temperature and wait time. The illustrated differences between the dynamics of a particle system and an archetypical spin glass can be assigned to the wide distribution of particle relaxation times and that some particles may relax independently of the collective spin-glass phase due to sample inhomogeneities.

The relaxation in the ac susceptibility, after cooling the sample to $23 \mathrm{~K}$ with the same cooling rate as in the reference measurement, is shown in Fig. 3. The relaxation in absolute units is larger for $\chi^{\prime}$ than for $\chi^{\prime \prime}$ and, in contrast to spin glasses $^{7}$ where the aging phenomena are best exposed in $\chi^{\prime \prime}$, the effects of aging are more clearly seen in $\chi^{\prime}$ for this particle system. Measuring the ac susceptibility with slower cooling rates gives lower values of the ac susceptibility. The effect of a slower cooling rate is largest just below the transition temperature where the effect of aging also is largest.

\section{B. Temperature cycling}

One experimental procedure that has been used for spin glasses to confirm the overlap length concept is temperature cycling. ${ }^{18}$ The measurements are similar to conventional aging experiments, but after the wait time $t_{\mathrm{w}_{1}}$ at the measuring temperature $T_{\mathrm{m}}$, a temperature change $\Delta T$ is made, and the system is exposed to a second wait time $t_{\mathrm{w}_{2}}$ before changing

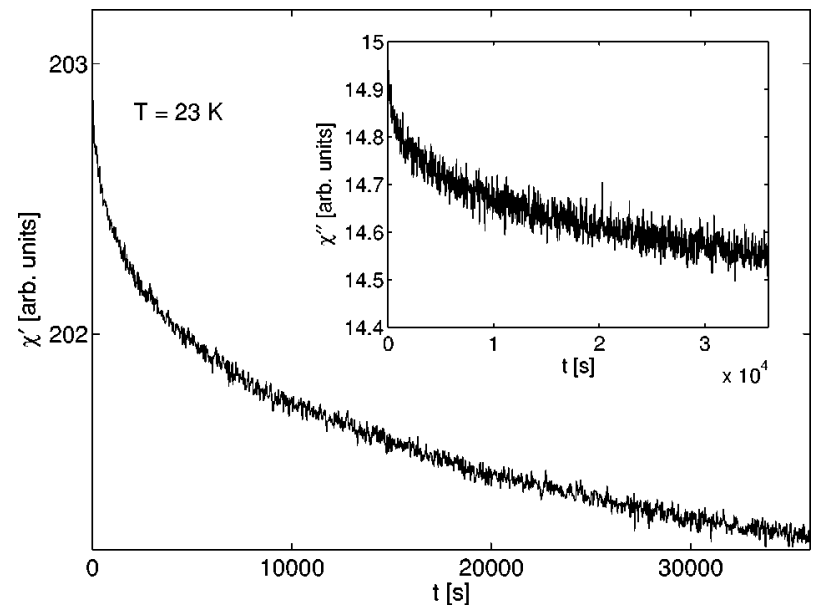

FIG. 3. ac susceptibility vs time at $T=23 \mathrm{~K}$. Same units as in Fig. $1, f=510 \mathrm{mHz}$.

the temperature back to $T_{\mathrm{m}}$, where the field is applied and the magnetization is recorded as a function of time.

Figure 4 shows results from measurements with negative temperature cycling, at $T_{\mathrm{m}}=30 \mathrm{~K}$, using $t_{\mathrm{w}_{1}}=3000$ and $t_{\mathrm{w}_{2}}=10000 \mathrm{~s}$. For $0>\Delta T \gtrsim-2 \mathrm{~K}$ the additional wait time at the cycling temperature mainly shifts the maximum in the relaxation rate to longer times. The shift is largest for the smallest $\Delta T$ and the maximum returns continuously towards $t_{\mathrm{w}_{1}}$ as $\Delta T$ is increased. For $\Delta T=-2 \mathrm{~K}$, the maximum appears at $\ln t \approx \ln t_{\mathrm{w}_{1}}$ and the relaxation rate behaves rather similar to the curve without the cycling only showing a somewhat larger magnitude (see Fig. 4). For a larger temperature step, $\Delta T=-4 \mathrm{~K}$, the relaxation rate is enhanced at times shorter than $t_{\mathrm{w}_{1}}$, while the relaxation appears unaffected at longer time scales. The behavior can be interpreted in terms of an interplay between the domain growth and the overlap length: for small temperature steps, the domain size $R_{1}\left(T_{\mathrm{m}}, t_{\mathrm{w}_{1}}\right)$ attained during $t_{\mathrm{w}_{1}}$ at $T_{m}$ is shorter than the

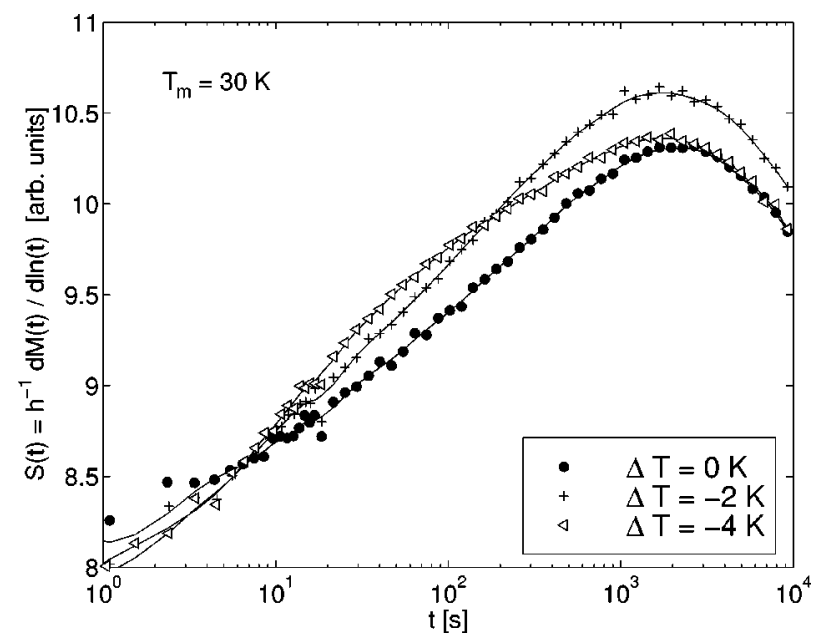

FIG. 4. Relaxation rate $S(t)$ vs time. The sample has been aged $t_{\mathrm{w}_{1}}=3000 \mathrm{~s}$ at $T_{\mathrm{m}}=30 \mathrm{~K}$ and afterward subjected to a negative temperature cycling $\Delta T$ during $t_{\mathrm{w}_{2}}=10000 \mathrm{~s}$, immediately prior to the application of the field $H=0.05$ Oe; circles: $\Delta T=0, t_{w_{2}}=0$; plus signs $\Delta T=-2 \mathrm{~K}$; triangles: $\Delta T=-4 \mathrm{~K}$. 


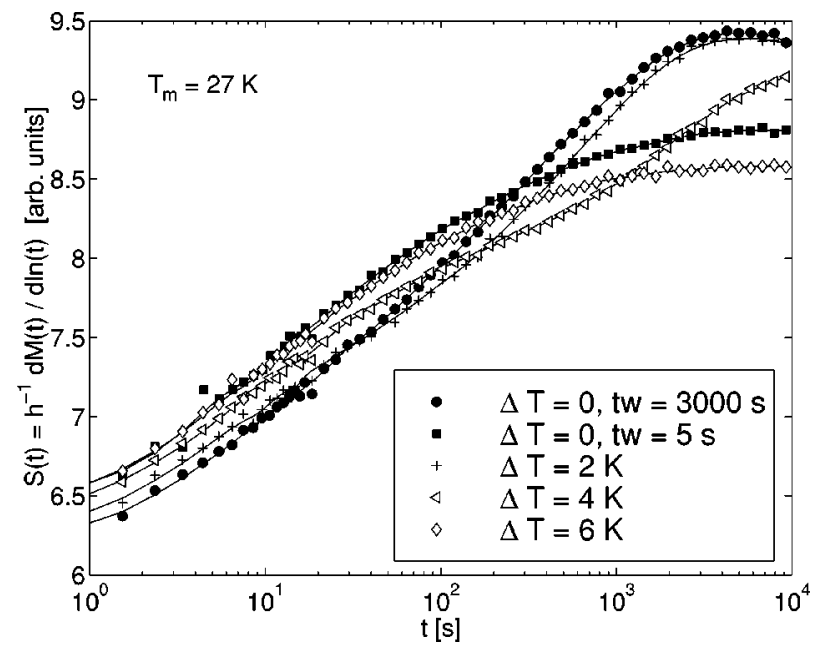

FIG. 5. Relaxation rate $S(t)$ vs time. The sample has been aged $t_{\mathrm{w}_{1}}=3000 \mathrm{~s}$ at $T_{\mathrm{m}}=27 \mathrm{~K}$ and afterward subjected to a positive temperature cycling $\Delta T$ during a time $t_{\mathrm{w}_{2}}$, immediately prior to the application of the field $H=0.05$ Oe; circles: $\Delta T=0$; plus signs: $\Delta T=2 \mathrm{~K}, t_{\mathrm{w}_{2}}=5 \mathrm{~s}$; triangles: $\Delta T=4 \mathrm{~K}, t_{\mathrm{w}_{2}}=5 \mathrm{~s}$; diamonds: $\Delta T=6 \mathrm{~K}, t_{\mathrm{w}_{2}}=30 \mathrm{~s}$. Squares are data obtained after the sample has been aged at $27 \mathrm{~K}$ for $5 \mathrm{~s}$ only.

overlap length at the cycling temperature and the domain growth proceeds essentially unaffected by the temperature change, only with a slower rate. For sufficiently large temperature steps the overlap length is shorter than $R_{1}\left(T_{\mathrm{m}}, t_{\mathrm{w}_{1}}\right)$ and the domain growth at the lower temperature creates a new domain structure on short length scales, $R_{2}\left(T_{\mathrm{m}}\right.$ $\left.+\Delta T, t_{\mathrm{w}_{2}}\right)$. For the temperatures and wait times used in the experiments $R_{2}\left(T_{\mathrm{m}}+\Delta T, t_{\mathrm{w}_{2}}\right)<R_{1}\left(T_{\mathrm{m}}, t_{\mathrm{w}_{1}}\right)$. Returning to $T_{\mathrm{m}}$, the new $R_{2}$ structures do not overlap with the equilibrium configuration and yield the apparent nonequilibrium nature of the dynamics on short time scales (cf. Fig. 4 for $\Delta T$ $=-4 \mathrm{~K})$.

Measurements with positive temperature cycling with $T_{\mathrm{m}}=27 \mathrm{~K}$ are shown in Fig. 5. For small temperature steps $(\Delta T \lesssim 2 \mathrm{~K})$ the maximum of the relaxation rate remains at $\ln t \approx \ln t_{\mathrm{w}_{1}}$, but for larger temperature steps it is seen that the relaxation rate approaches the relaxation rate of an aging measurement with short wait time. This can again be explained by the overlap length becoming shorter than $R_{1}\left(T_{\mathrm{m}}, t_{\mathrm{w}_{1}}\right)$ when $\Delta T \gtrsim 2 \mathrm{~K}$. Since the domain growth rate increases fast with increasing temperature, the system will then look more and more reinitialized when returning to $T_{\mathrm{m}}$ after larger $\Delta T$ and/or longer $t_{\mathrm{w}_{2}}$.

\section{Memory}

The experimental procedure for a memory experiment is illustrated in Fig. 6. $\chi^{\prime}(T)$ is recorded during cooling, employing different halts at constant temperature, followed by continuous heating. Figure 7 shows results from three different memory experiments: the first with a single temporary halt at $33 \mathrm{~K}$ for $1 \mathrm{~h}$ and $30 \mathrm{~min}$, the second with a single temporary halt at $23 \mathrm{~K}$ for $10 \mathrm{~h}$, and the third with temporary halts at both 33 and $23 \mathrm{~K}$. The exposed curves show the difference between the measured and a reference curve. Ref-

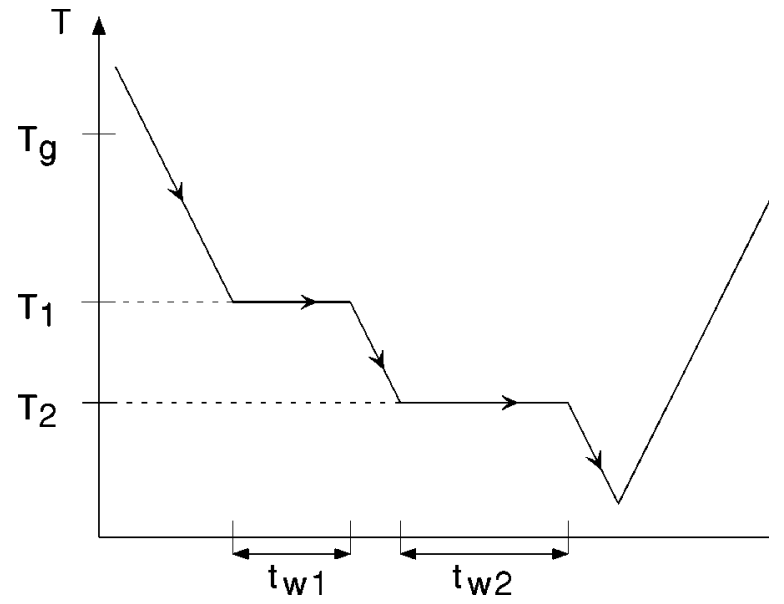

FIG. 6. The experimental procedure in an ac-susceptibility memory experiment with two temporary halts during cooling.

erence curves are taken from a measurement with continuous cooling followed by continuous heating. Similar to spin glasses, a memory of the cooling history is observed on heating for both one and two temporary halts. It is seen that on the low-temperature side, the susceptibility approaches the reference level slower than on the high-temperature side. This asymmetry of the dip is more pronounced in the particle system than for spin glasses. The relaxation at $23 \mathrm{~K}$ after a temporary halt at $33 \mathrm{~K}$ is equally large as the relaxation at 23 $\mathrm{K}$ without earlier halt, except that it starts at a lower level. In fact, $\chi^{\prime}-\chi_{\text {ref }}^{\prime}$, measured on heating for the experiment with two temporary halts at 33 and $23 \mathrm{~K}$, is just the sum of $\chi^{\prime}$ $-\chi_{\text {ref }}^{\prime}$ of the two experiments with a single temporary halt at 33 and $23 \mathrm{~K}$, respectively.

In Fig. 8 is shown a memory experiment similar to the one in Fig. 7 except that the two temporary halts are closer in temperature. The ac susceptibility has been measured with temporary halts on cooling at $33 \mathrm{~K}$ for $1 \mathrm{~h} 30 \mathrm{~min}$ and at 28 $\mathrm{K}$ for $7 \mathrm{~h}$. On heating, the double halt experiment only shows

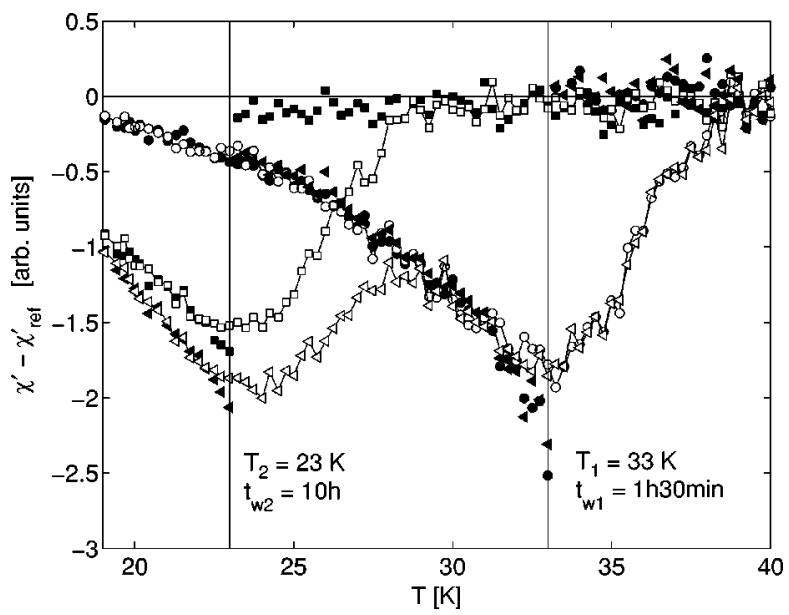

FIG. 7. $\chi^{\prime}(T)-\chi_{\text {ref }}^{\prime}(T)$ vs $T$ measured while cooling (solid symbols) or heating (open symbols). Circles: the cooling was halted at $33 \mathrm{~K}$ for $1 \mathrm{~h} 30 \mathrm{~min}$; squares: the cooling was halted at $23 \mathrm{~K}$ for $10 \mathrm{~h}$; triangles: the cooling was halted at $T_{1}=33 \mathrm{~K}$ for $t_{\mathrm{w}_{1}}=1 \mathrm{~h} 30$ $\min$ and at $T_{2}=23 \mathrm{~K}$ for $t_{\mathrm{w}_{1}}=10 \mathrm{~h}$. Units and frequency are the same as in Fig. 1. 


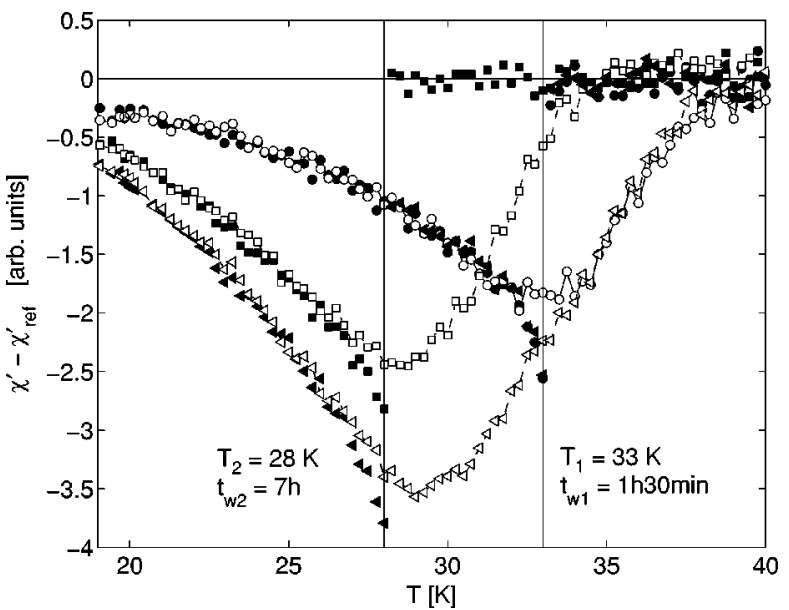

FIG. 8. $\chi^{\prime}(T)-\chi_{\text {ref }}^{\prime}(T)$ vs $T$ measured while cooling (solid symbols) or heating (open symbols). Circles: the cooling was halted at $33 \mathrm{~K}$ for $1 \mathrm{~h} 30 \mathrm{~min}$; squares: the cooling was halted at $28 \mathrm{~K}$ for $7 \mathrm{~h}$; triangles: the cooling was halted at $T_{1}=33 \mathrm{~K}$ for $t_{\mathrm{w}_{1}}=1 \mathrm{~h} 30$ min and at $T_{2}=28 \mathrm{~K}$ for $t_{\mathrm{w}_{1}}=7 \mathrm{~h}$. Units and frequency are the same as in Fig. 1.

one dip. However, this $\operatorname{dip}\left(\chi^{\prime}-\chi_{\text {ref }}^{\prime}\right)$ still constitutes the sum of the two heating curves with one halt, as was the case for the experiments shown in Fig. 7. Also for this experiment, the ac-relaxation curve at the lower temperature is the same with and without temporary halt at $33 \mathrm{~K}$, except for a constant.

We have performed supplementary experiments to the ones shown in Figs. 7 and 8 by measuring the dc relaxation. In Fig. 9, the relaxation rate of three dc-relaxation curves measured at $33 \mathrm{~K}$ are shown. In the three experiments the system was quenched to $33 \mathrm{~K}$ and then aged for $1 \mathrm{~h} 30 \mathrm{~min}$. In one of the measurements the relaxation was measured immediately after this wait time, but in the two other measurements a negative temperature cycling was performed to $23 \mathrm{~K}$ for $10 \mathrm{~h}$ and to $28 \mathrm{~K}$ for $7 \mathrm{~h}$, respectively. The dynamics is sizeably affected by the temperature cycling to $28 \mathrm{~K}$, but only weakly affected by the temperature cycling to $23 \mathrm{~K}$.

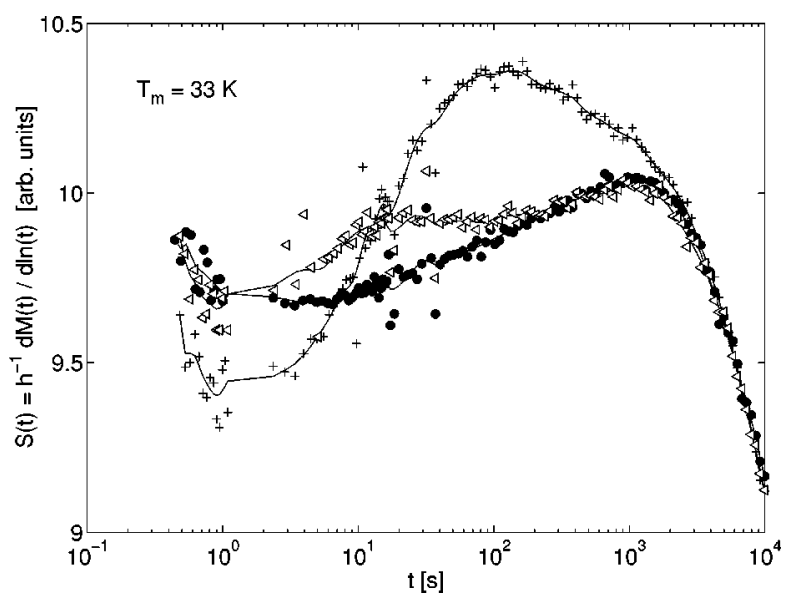

FIG. 9. Relaxation rate $S(t)$ vs time. The sample has been aged for $1 \mathrm{~h} 30 \mathrm{~min}$ at $T_{\mathrm{m}}=33 \mathrm{~K}$ and afterward subjected to a negative temperature cycling $\Delta T$ during $t_{\mathrm{w}_{2}}$. Circles: $\Delta T=0$; plus signs: $T_{\mathrm{m}}+\Delta T=28 \mathrm{~K}, \quad t_{\mathrm{w}_{2}}=7 \mathrm{~h} ;$ triangles: $T_{\mathrm{m}}+\Delta T=23 \mathrm{~K}, t_{\mathrm{w}_{2}}$ $=10$ h. $H=0.05$ Oe.

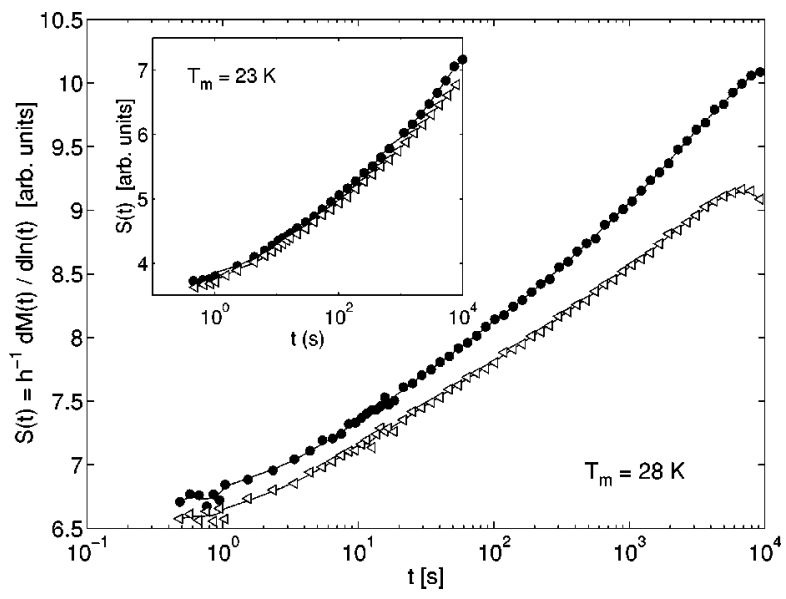

FIG. 10. Relaxation rate $S(t)$ vs time. The sample has been aged, after a quench, for $t_{\mathrm{w}}=7 \mathrm{~h}$ at $T_{\mathrm{m}}=28 \mathrm{~K}$ (circles) and after a quench with a temporary halt at $33 \mathrm{~K}$ for $1 \mathrm{~h} 30 \mathrm{~min}$ (triangles), $H=0.05$ Oe. Inset: $T_{\mathrm{m}}=23 \mathrm{~K}$ and $t_{\mathrm{w}}=10 \mathrm{~h}$.

The observed increase of the relaxation rate indicates that the equilibrium structure created at $33 \mathrm{~K}$ is erased on short length scales after the temperature cycling, as discussed in Sec. IV B. On time scales shorter than $10 \mathrm{~s}, S(t)$ is even lower for the experiment with a temperature cycling to $28 \mathrm{~K}$ than for the other two experiments. We conclude that there is an overlap between the equilibrium configuration of the magnetic moments at 33 and $28 \mathrm{~K}$ on length scales shorter than $L(33 \mathrm{~K}, 10 \mathrm{~s})$. Let us now compare these dc measurements with the corresponding ac measurements. The inphase component of the ac susceptibility at a frequency of $510 \mathrm{mHz}$ records the integrated response corresponding to observation times $1 / \omega \approx 0.3 \mathrm{~s}$ and shorter. In the ac experiment with two temporary halts at 33 and at $23 \mathrm{~K}$ (Fig. 7), no effect of the aging at $23 \mathrm{~K}$ is seen when heating through 33 $\mathrm{K}$. However, $\chi^{\prime}(33 \mathrm{~K})$ measured on heating for the ac measurement with temporary halts at 33 and $28 \mathrm{~K}$ is lower than $\chi^{\prime}(33 \mathrm{~K})$ for the measurement with only a single halt at 33 $\mathrm{K}$. A lower level of $\chi^{\prime}(T)$ indicates a more equilibrated system and thereby also a lower relaxation rate. This supports the above conclusion of some overlap between the equilibrium configurations at 28 and $33 \mathrm{~K}$ on the length scales seen by observation times shorter than $0.3 \mathrm{~s}$.

Figure 10 shows the relaxation rate measured at 28 and 23 $\mathrm{K}$ after a quench to $T_{\mathrm{m}}$ followed by a wait time of 7 and 10 $\mathrm{h}$, respectively. The same measurements except for a temporary halt at $33 \mathrm{~K}$ for $1 \mathrm{~h} 30 \mathrm{~min}$ are also shown. The relaxation rate is lower for the measurement with a temporary halt at $33 \mathrm{~K}$ for both temperatures, even though the difference between a temporary halt and a direct quench is larger at 28 $\mathrm{K}$. These measurements, as the corresponding ac measurements, show that there is some overlap on short length scales between the equilibrium configuration at $33 \mathrm{~K}$ and all lower temperatures for which we have measured the ac susceptibility.

\section{CONCLUSIONS}

The nonequilibrium dynamics in the low-temperature spin-glass-like phase of an interacting $\mathrm{Fe}-\mathrm{C}$ nanoparticle sample is found to largely mimic the corresponding spin- 
glass dynamics. The observed differences may be accounted for by the strongly temperature-dependent and widely distributed relaxation times of the particle magnetic moments compared to the temperature-independent and monodispersed relaxation times of the spins in a spin glass. Within a droplet scaling picture of a particle system, these factors strongly affects the associated length scales and growth rates of the domains and droplet excitations as well as the roughness of the domain walls. In addition, there might exist individual particles that do not take part in the collective lowtemperature spin-glass phase but relax independently.

This work was financially supported by The Swedish Natural Science Research Council (NFR).
${ }^{1}$ C. Djurberg, P. Svedlindh, P. Nordblad, M. F. Hansen, F. Bødker, and S. Mørup, Phys. Rev. Lett. 79, 5154 (1997).

${ }^{2}$ T. Jonsson, P. Svedlindh, and M. F. Hansen, Phys. Rev. Lett. 81, 3976 (1998).

${ }^{3}$ H. Mamiya, I. Nakatani, and T. Furubayashi, Phys. Rev. Lett. 80, 177 (1998).

${ }^{4}$ P. Nordblad and P. Svedlindh, in Spin Glasses and Random Fields, edited by A. P. Young (World Scientific, Singapore, 1998), pp. 1-27.

${ }^{5}$ T. Jonsson, J. Mattsson, C. Djurberg, F. A. Khan, P. Nordblad, and P. Svedlindh, Phys. Rev. Lett. 75, 4238 (1995).

${ }^{6}$ H. Mamiya, I. Nakatani, and T. Furubayashi, Phys. Rev. Lett. 82, 4332 (1999).

${ }^{7}$ K. Jonason, E. Vincent, J. Hammann, J. P. Bouchaud, and P. Nordblad, Phys. Rev. Lett. 81, 3243 (1998).

${ }^{8}$ T. Jonsson, K. Jonason, P. Jönsson, and P. Nordblad, Phys. Rev. B 59, 8770 (1999).

${ }^{9}$ K. Jonason, P. Nordblad, E. Vincent, J. Hammann, and J. P.
Bouchaud, cond-mat/9904410, Eur. Phys. J. B (to be published).

${ }^{10}$ C. Djurberg, K. Jonason, and P. Nordblad, Eur. Phys. J. B 10, 15 (1999).

${ }^{11}$ P. Jönsson, M. F. Hansen, P. Svedlindh, and P. Nordblad, Physica B (to be published).

${ }^{12}$ D. S. Fisher and D. A. Huse, Phys. Rev. B 38, 373 (1988); 38, 386 (1988).

${ }^{13}$ L. Lundgren, P. Svedlindh, and O. Beckman, J. Magn. Magn. Mater. 25, 33 (1981)

${ }^{14}$ J. Van Wonterghem, S. Mørup, S. W. Charles, and S. Wells, J. Colloid Interface Sci. 121, 558 (1988).

${ }^{15}$ M. F. Hansen, F. Bødker, S. Mørup, C. Djurberg, and P. Svedlindh, J. Magn. Magn. Mater. 177-181, 928 (1998).

${ }^{16}$ M. F. Hansen, P. Jönsson, P. Nordblad, and P. Svedlindh (unpublished).

${ }^{17}$ J. Magnusson, C. Djurberg, P. Granberg, and P. Nordblad, Rev. Sci. Instrum. 68, 3761 (1997).

${ }^{18}$ L. Sandlund, P. Svedlindh, P. Granberg, P. Nordblad, and L. Lundgren, J. Appl. Phys. 64, 5616 (1988). 